\title{
Effect of Surface Tension on Nanocontact Problem
}

\author{
Liyuan Wang \\ Longqiao College of Lanzhou University of Finance and Economics, Lanzhou 730101, China
}

\begin{abstract}
This paper proposes an application of surface elasticity theory in the analysis of contact problem at nano-scale. The Fourier integral transform method is adopted to derive the fundamental solutions for contact problem with surface tension effects. As a special case, the deformation induced by normal triangle distribution force is discussed in detail. The results indicate some interesting characteristics in nano-mechanics, which are distinctly different from those in classical contact problem. The results show that the hardness of material depends strongly on the surface tension.
\end{abstract}

Keywords-surface tension; nano-contact problem; normal triangle distribution force; fourier integral transform method

\section{INTRODUCTION}

The surface of solids is a special region with very small thickness (a few times of atom-spacing). Since the equilibrium lattice spacing in the surface is different from that in the bulk, surface effect appears. For solids with large characteristic dimensions, the volume ratios of surface region to the bulk material is small, the effect of surface then can be neglected because of its relatively tiny contribution. However, for micronano solids with large surface-to-bulk ratio the significance of surfaces is likely to be important. Form the viewpoint of continuum mechanics, this difference can be described by such concepts as surface tension, surface energy, and surface constitutive relations [1]. This is extremely true for nano-scale materials or structures. In such cases, the surface residual tension plays a critical role and thus has been adding its appeal to many researchers. For example, Miller and Shenoy [2] first probed the size-dependent elastic properties of nanoplates and beams. Hang et al. [3] explained the size dependent phenomenon by the strain gradient continuum theory. Sharma et al. [4] and Sharma and Gantin [5] studied the effect of surface/interfacial energy on the Eshelby's inclusion and inhomogeneity problems. Dingreville et al. [6] investigated the surface free energy and its effect on elastic behavior of the nanosized particles, wires, and films. There are a lot of work regarding the surface/interface energy effects on the nanostructures and solids, and we can only include a small part of them here. For more recent developments in this field, the readers can refer to a review article by Wang et al. [7].

To study the mechanical behavior of an immediate neighborhood of material surfaces through a continuum-based model, Gurtin and Murdoch [8,9] developed a mathematical framework, known as the theory of surface elasticity. In the study of nano-scale problems, all material constants appearing in that constitutive model were commonly calibrated with data obtained from either experimental measurements [10] or atomistic simulations $[2,11]$. Therefore, the surface effect has been widely adopted to investigate the mechanical phenomena at nano-scale. Wang et al. [12] studied the response of a halfplane subjected to normal pressures with constant residual surface tension. Long and Wang [13] studied the effect of the residual surface stress on the two dimensional Hertz contact problem, and later Long et al. [14] generalized their work to the three dimensional case. Wang [15] derived the general analytical solution of nano-contact problem with surface effects by using the complex variable function method. In this paper, Fourier integral transform method is used to solve the nonclassical boundary value problems with surface tension effects.

\section{BASIC EQUATIONS}

In the absence of body force, the equilibrium equations, constitutive law, and geometry relations in the bulk are as follows

$$
\begin{aligned}
& \sigma_{i j, j}=0 \\
& \sigma_{i j}=2 G\left(\varepsilon_{i j}+\frac{\mu}{1-2 \mu} \varepsilon_{k k} \delta_{i j}\right)
\end{aligned}
$$

where $\mathrm{G}$ and $\mu$ are the shear modulus and Poisson's ratio of the bulk material, $\sigma_{i j}$ and $\varepsilon_{i j}$ are the stress tensor and strain tensor in the bulk material, respectively. Throughout the paper, Einstein's summation convention is adopted for all repeated Latin indices $(1,2,3)$ and Greek indices $(1,2)$.

The strain tensor is related to the displacement vector $u_{i}$ by

$$
\varepsilon_{i j}=\frac{1}{2}\left(u_{i, j}+u_{j, i}\right)
$$

On the surface, the generalized Young-Laplace equation, surface constitutive relation and strain-displacement relationship can be expressed as

$$
\begin{gathered}
\sigma_{\beta \alpha} n_{\beta}+\sigma_{\beta \alpha, \beta}^{s}=0 \\
\sigma_{i j} n_{i} n_{j}=\sigma_{\beta \alpha}^{s} \kappa_{\beta \alpha} \\
\sigma_{\beta \alpha}^{s}=\tau^{s} \delta_{\beta \alpha}+\frac{\partial \tau^{s}}{\partial \varepsilon_{\beta \alpha}}
\end{gathered}
$$


where $n_{i}$ denotes the normal to the surface, $\kappa_{\beta \alpha}$ the curvature tensor of the surface, $\sigma_{\alpha \beta}^{s}$ and $\varepsilon_{\alpha \beta}^{s}$ the surface stress and surface strain tensor, $\tau^{s}$ is the residual surface tension under unstrained conditions.

\section{GeNerAl SOLUTIONS}

Now we consider a material occupying the upper halfplane $Z>0$, we refer to a Cartesian coordinate system (o-xyz), where the $x$ axis is along the surface and the $z$ axis perpendicular to the surface. It is assumed that the material is subjected to a normal triangle distribution force $p(x)$ over the region $|x| \leq a$. While the normal forces maximum $p_{0}$ at the point $O$. The contact is assumed to be frictionless.

After For the considered plane problem, the equilibrium equations and Hooke's law in the bulk reduce to

$$
\begin{aligned}
& \frac{\partial \sigma_{x x}}{\partial x}+\frac{\partial \sigma_{x z}}{\partial z}=0, \frac{\partial \sigma_{z z}}{\partial z}+\frac{\partial \sigma_{x z}}{\partial x}=0 \\
& \varepsilon_{x x}=\frac{1}{2 G}\left[(1-v) \sigma_{x x}-v \sigma_{z z}\right] \\
& \varepsilon_{z z}=\frac{1}{2 G}\left[(1-v) \sigma_{z z}-v \sigma_{x x}\right] \\
& \varepsilon_{x x}=\frac{\sigma_{x z}}{2 G}
\end{aligned}
$$

The strains are related to the displacements by

$$
\varepsilon_{x x}=\frac{\partial u}{\partial x}, \varepsilon_{z z}=\frac{\partial w}{\partial z}, \varepsilon_{x z}=\frac{1}{2}\left(\frac{\partial u}{\partial z}+\frac{\partial w}{\partial x}\right)
$$

which satisfy the following compatibility condition

$$
\frac{\partial^{2} \varepsilon_{x x}}{\partial z^{2}}+\frac{\partial^{2} \varepsilon_{z z}}{\partial x^{2}}=2 \frac{\partial^{2} \varepsilon_{x z}}{\partial x \partial z} .
$$

As in classical theory of elasticity, the Airy stress function $\chi(x, z)$ is defined by

$$
\sigma_{x x}=\frac{\partial^{2} \chi}{\partial z^{2}}, \sigma_{z z}=\frac{\partial^{2} \chi}{\partial x^{2}}, \sigma_{x z}=-\frac{\partial^{2} \chi}{\partial x \partial z}
$$

Then the equilibrium equations in Eq. (7) are satisfied automatically, and the compatibility equation in Eq. (8) becomes

$$
\left(\frac{\partial^{2}}{\partial x^{2}}+\frac{\partial^{2}}{\partial z^{2}}\right)\left(\frac{\partial^{2} \chi}{\partial x^{2}}+\frac{\partial^{2} \chi}{\partial z^{2}}\right)=0
$$

To solve the boundary value problem, the Fourier integral transformation method is adopted to the coordinate $x$. Then, the Airy stress function $\chi(x, z)$ and its Fourier transformation $\tilde{\chi}(\xi, z)$ can be expressed as

$$
\begin{aligned}
& \tilde{\chi}(\xi, z)=\frac{1}{\sqrt{2 \pi}} \int_{-\infty}^{\infty} \chi(x, z) e^{i x \xi} d \xi \\
& \chi(\xi, z)=\frac{1}{\sqrt{2 \pi}} \int_{-\infty}^{\infty} \tilde{\chi}(x, z) e^{-i x \xi} d \xi
\end{aligned}
$$

Substituting Eqs. (10) into Eq. (9) and considering the condition that the stresses vanish at infinity, one obtains

$$
\tilde{\chi}(\xi, z)=(A+B z) e^{-z|\xi|}
$$

where $A$ and $B$ are generally functions of $\xi$ as yet to be determined by boundary conditions.

Substituting Eq. (11) and Eqs. (10) into Eq. (9), the stresses can be written as

$$
\begin{aligned}
& \sigma_{x x}=\frac{1}{\sqrt{2 \pi}} \int_{-\infty}^{\infty}[A(\xi)+(z-2|\xi|) B(\xi)] \xi e^{-i x \xi-z|\xi|} d \xi \\
& \sigma_{z z}=-\frac{1}{\sqrt{2 \pi}} \int_{-\infty}^{\infty}[A(\xi)+z B(\xi)] \xi^{2} e^{-i x \xi-z|\xi|} d \xi \\
& \sigma_{x z}=\frac{i}{\sqrt{2 \pi}} \int_{-\infty}^{\infty} \xi[(1-|\xi| z) B(\xi)-|\xi| A(\xi)] e^{-i x \xi-z|\xi|} d \xi
\end{aligned}
$$

By substituting the stresses into the Eq. (9) and using Eqs. (10), the displacements are derived as

$$
\begin{aligned}
& u(x, z)=\frac{i}{2 G \sqrt{2 \pi}} \int_{-\infty}^{\infty}[(2-v)|\xi| A(\xi)+(z|\xi|-2(1-v)) B(\xi)] e^{-i k \xi-2|\xi|} d \xi+C_{1} \\
& w(x, z)=\frac{1}{2 G \sqrt{2 \pi}} \int_{-\infty}^{\infty}[|\xi| A(\xi)+(1-2 v+z|\xi|) B(\xi)] e^{-\alpha-\xi \xi-|| \xi \mid} d \xi+C_{2}
\end{aligned}
$$

\section{Elastic SOlution Under a NoRmal TRIANGLE DISTRIBUTION FORCE}

As a particular example, let us consider the effect of a normal triangle distribution force $p(x)$ over the region $|x| \leq a$, while remainder of the boundary $y=0$ being unstressed.

$$
p(x)=\frac{p_{0}}{a}(a-|x|),|x| \leq a
$$


Due to the second term in Eq. (4) indicates a variation of the surface energy density with respect to elastic strain, which is related to the stretching or compressing the atoms in the surface to accommodate to the bulk phase. If the change of the atomic spacing in deformation is infinitesimal, the contribution from the second term to the surface stresses is negligibly small compared to the residual surface tension [8]. In what follows, we keep only the first term in Eq. (4). Then, the surface stresses are given by

$$
\sigma_{\beta \alpha}^{s}=\tau^{s} \delta_{\beta \alpha}
$$

In this case, the boundary conditions (3) on the contact surface $(Z=0)$ are simplified to

$$
\begin{aligned}
& \sigma_{x z}(x)=0 \\
& p(x)+\sigma_{z z}(x)=-\frac{\tau^{s}}{R(x)}
\end{aligned}
$$

Substituting Eqs. (16) into Eqs. (12), one obtains

$$
B=A|\xi|
$$

On the surface, the radius of curvature due to deformation is given by

$$
1 / R(x)=\partial^{2} w(x, 0) / \partial x^{2}
$$

By substituting Eqs. (17) and (18) into the surface condition Eqs. (16), $A(\xi)$ is determined by

$$
A(\xi)=\frac{\tilde{p}(\xi)}{(1+s|\xi|) \xi^{2}}
$$

where

$$
s=\frac{\tau^{s}(1-v)}{G}, \tilde{p}(\xi)=\frac{p_{0}}{a} \sqrt{\frac{2}{\pi}} \frac{1-\cos (a \xi)}{\xi^{2}}
$$

Therefore $A(\xi)$ is given by

$$
A(\xi)=\frac{p_{0}}{a} \sqrt{\frac{2}{\pi}} \frac{1-\cos (a \xi)}{(1+s|\xi|) \xi^{4}}
$$

Substituting Eq. (21) into Eqs. (12) and (13), the stresses component and displaces component are obtained as

$$
\begin{aligned}
& \sigma_{x x}=\frac{2 q_{0}}{\pi a} \int_{0}^{\infty}\left(\frac{z \xi-1}{1+s \xi}\right) \frac{\cos (x \xi)}{\xi^{2}}[1-\cos (a \xi)] e^{-z \xi} d \xi \\
& \sigma_{z z}=-\frac{2 q_{0}}{\pi a} \int_{0}^{\infty}\left(\frac{z \xi+1}{1+b \xi}\right) \frac{\cos (x \xi)}{\xi^{2}}[1-\cos (a \xi)] e^{-z \xi} d \xi \\
& \sigma_{x z}=-\frac{2 q_{0}}{\pi a} \int_{0}^{\infty}\left(\frac{z}{1+s \xi}\right) \frac{\sin (x \xi)}{\xi}[1-\cos (a \xi)] e^{-z \xi} d \xi \\
& u(x, z)=\frac{p_{0}}{\pi G a} \int_{0}^{\infty}\left[\frac{z \xi+2 v-1}{\xi^{3}(1+s \xi)}\right] \sin (x \xi)[1-\cos (a \xi)] e^{-z \xi} d \xi+C_{1} \\
& w(x, z)=\frac{p_{0}}{\pi G a} \int_{0}^{\infty} \frac{z \xi+2(1-v)}{\xi^{3}(1+b \xi)} \cos (x \xi)[1-\cos (a \xi)] e^{-z \xi} d \xi+C_{2}
\end{aligned}
$$

It is seen, when $s=0$, that is, the surface influence is ignored in Eqs. (22), the stresses of the half-plane are consistent with those in the classical elastic results [16].

On the contact surface $Z=0$, the normal stress is given by

$$
\sigma_{z z}(x, 0)=-\frac{2 p_{0}}{\pi} \int_{0}^{\infty}\left(\frac{1-\cos t}{t^{2}}\right)\left(1+\frac{s}{a} t\right)^{-1} \cos \left(\frac{x}{a} t\right) d t
$$

If the normal displacement is $w$ specified to be zero at a distance $r_{0}$ on the contact surface, that is, $w\left(r_{0}, 0\right)=0$, the displacement on the surface is derived as

$$
w(x, 0)=\frac{2 p_{0}(1-v) a}{\pi G} \int_{0}^{\infty}\left(\frac{1-\cos (t)}{t^{3}}\right)\left(\frac{s}{a}+1\right)^{-1}\left[\cos \left(\frac{x}{a} t\right)-\cos \left(\frac{r_{0}}{a} t\right)\right] d t
$$

As show in Figure 1, the distribution of the normal stress $\sigma_{\mathrm{zz}}$ that is predicted by the classical theory experiences a singularity at the loading boundary and does not change smoothly; thus, this result appears unreasonable. The results that take into account the residual surface tension give a smooth distribution of the stress $\sigma_{\mathrm{zz}}$ and overcome the singularity at the loading boundary $x= \pm a$. If the residual surface tension is ignored ( $s / a=0$ ), the values of $\sigma_{\text {zz }}$ reduce to the classical values.

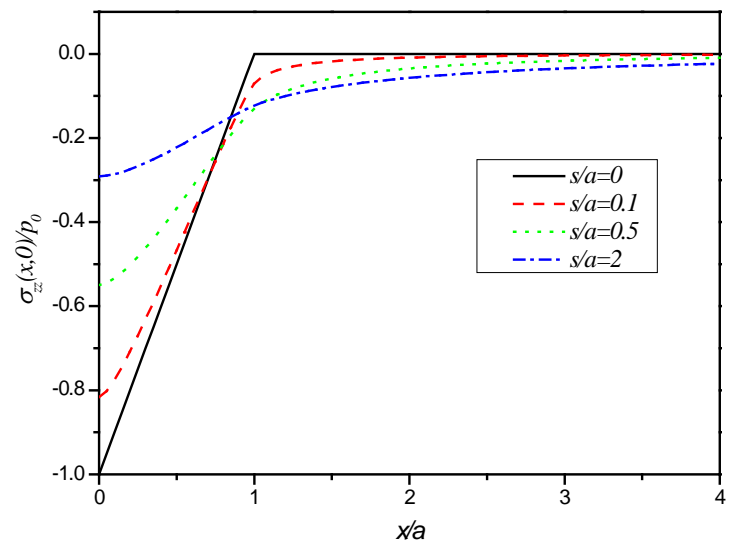

FIGURE I. THE DISTRIBUTION OF CONTACT NORMAL STRESS $\sigma_{\mathrm{zz}}$ 
Due to the different residual surface stress value, indent depth is plotted in Figure 2 with $K=2(1-v) / \pi G$, which also shows that the slope of the deformed surface for $a>0$ is continuous everywhere. It is also found the indent depth decreases with the increase of residual surface tension.

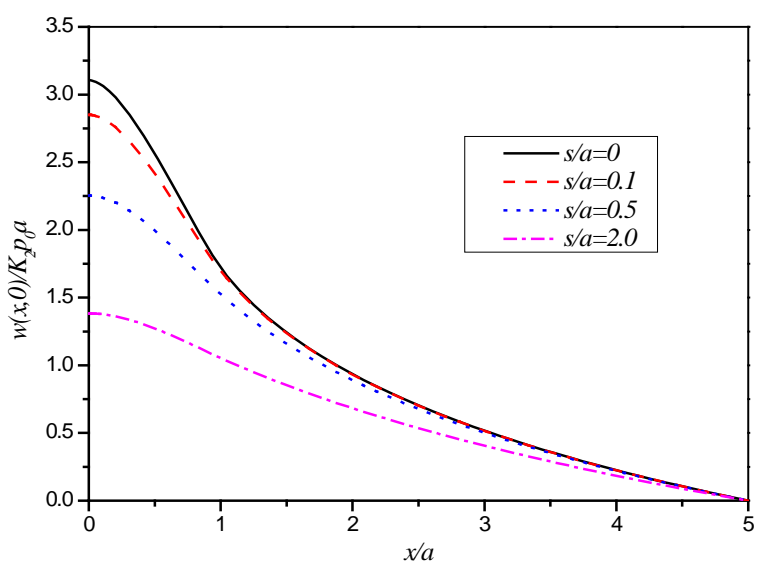

FIGURE II. THE DISTRIBUTION OF SURFACE INDENTATION $w$ UNDER NORMAL TRIANGLE DISTRIBUTION LOAD

To elucidate the size dependence of hardness on indenter size in nanoindentation, the following parameter is $H$ defined by

$$
H=\frac{2 p_{0} a}{w(0,0)}=\frac{\pi G}{1-v}\left[\int_{0}^{\infty}\left(\frac{1-\cos (t)}{t^{3}}\right)\left(\frac{s}{a}+1\right)^{-1}\left[1-\cos \left(\frac{r_{0}}{a} t\right)\right] d t\right]^{-1}
$$

To stand for the hardness of material subjected to the normal triangle distribution load. The variation of $\mathrm{H} / \mathrm{H}_{0}$ with respect to the indenter size $a$ is shown in Figure 3, where $H_{0}=\pi G /(1-v)$. The numerical results illustrate that the size effect becomes remarkable. At the nano-scale, the smaller the contact region, the larger the contact stiffness compared with the classical result.

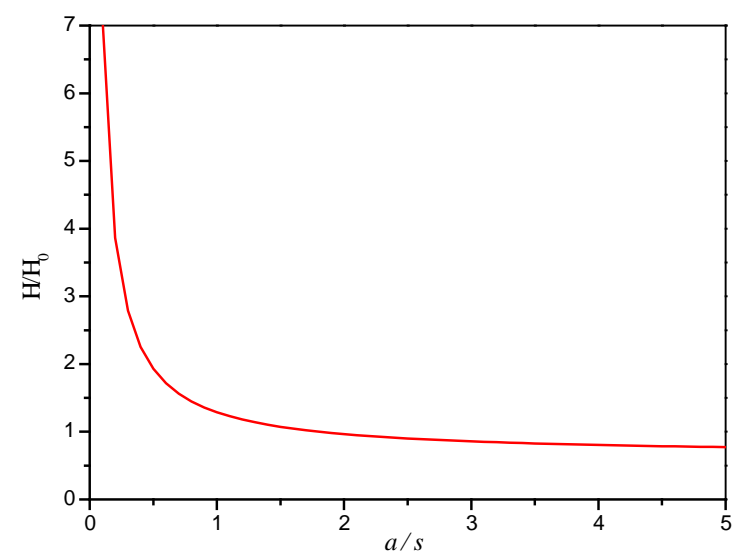

FIGURE III. THE VARIATION OF HARDNESS $H / H_{0}$ WITH RESPECT TO THE CONTACT ZONE SIZE

\section{CONCLUSIONS}

In this paper, we consider the two-dimensional contact problem in the light of surface elasticity theory. The general analytical solution is derived by using the Fourier integral transform method. For a particular loading case of normal triangle distribution force, the results are analyzed in detail and compared with the classical linear elastic solutions. A series of theoretical and numerical results show that the surface elasticity theory illuminates some interesting characteristics of contact problems at nano-scale, which are distinctly different from the classical solutions of elasticity without surface effects. Therefore the effects of surface tension should be considered for nanocontact problems.

\section{ACKNOWLEDGEMENTS}

This research was supported by the Higher Education Scientific Research Program of Gansun Province of China(No. 2017A251).

\section{REFERENCES}

[1] Cammarata, Robert C. "Surface and interface stress effects in thin films." Progress in Surface Science 46.1(1994):1-38.

[2] Miller, Ronald E., and V. B. Shenoy. "Size-dependent elastic properties of nanosized structural elements." Nanotechnology 11.3(2000):139-147

[3] Huang, Y., et al. "A model of size effects in nano-indentation." Journal of the Mechanics \& Physics of Solids 54.8(2006):1668-1686.

[4] Sharma, P., S. Ganti, and N. Bhate. "Effect of surfaces on the sizedependent elastic state of nano-inhomogeneities." Applied Physics Letters 82.4(2003):535-537.

[5] Sharma, P., and Ganti, S. "Size-Dependent Eshelby's Tensor for Embedded Nano-Inclusions Incorporating Surface/Interface Energies."Journal of Applied Mechanics 72.4(2005):663-671.

[6] Dingreville, Rémi, J. Qu, and M. Cherkaoui. "Surface free energy and its effect on the elastic behavior of nano-sized particles, wires and films."Journal of the Mechanics \& Physics of Solids 53.8(2005):18271854

[7] Jianxiang, et al. "SURFACE STRESS EFFECT IN MECHANICS OF NANOSTRUCTURED MATERIALS." Acta Mechanica Solida Sinica 24.1(2011):52-82.

[8] Gurtin, Morton E., and A. I. Murdoch. "A continuum theory of elastic material surfaces." Archive for Rational Mechanics \& Analysis 57.4(1975):291-323.

[9] Gurtin, Morton E., and A. I. Murdoch. "Surface stress in solids."International Journal of Solids \& Structures 14.6(1978):431-440.

[10] Jing, G. Y., et al. "Surface effects on elastic properties of silver nanowires: Contact atomic-force microscopy." Physical Review B 73.23(2006):235409.

[11] Shenoy, Vijay B. "Atomistic calculations of elastic properties of metallic fcc crystal surfaces." Physical Review B 71.9(2005):4104.

[12] Wang, G. F., and X. Q. Feng. "Effects of surface stresses on contact problems at nanoscale." Journal of Applied Physics 101.1(2007):013510-013510-6.

[13] Long, J. M., et al. "Two-dimensional Hertzian contact problem with surface tension." International Journal of Solids \& Structures49.13(2012):1588-1594.

[14] Long, J. M., and G. F. Wang. "Effects of surface tension on axisymmetric Hertzian contact problem." Mechanics of Materials56.1(2013):65-70.

[15] Wang, L. Y. "Elastic analysis for contact problems with surface effects under normal load." Mathematics \& Mechanics of Solids (2015).

[16] K. L. Johnson, Contact Mechanics (CambridgeUniversity Press, London, 1985. 